CERN-PPE/95-80

26 May 1995

\title{
NEW RESULTS FROM NA45/CERES
}

Peter Wurm for the CERES Collaboration:

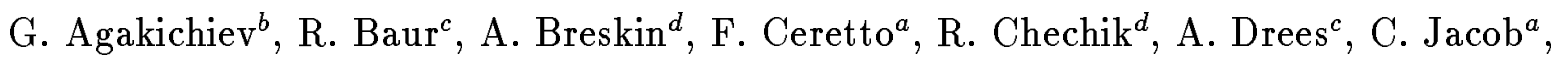
U. Faschingbauer ${ }^{a}$, P. Fischer ${ }^{c, 1}$, Z. Fraenkel ${ }^{d}$, Ch. Fuchs ${ }^{a}$, E. Gatti ${ }^{e}$, P. Glässel ${ }^{c}$, Th. Günzel ${ }^{c}$, C.P. de los $\operatorname{Heros}^{d}$, F. Hess ${ }^{a}$, D. Irmscher ${ }^{c}$, H. Kraner ${ }^{f}$, B. Lenkeit ${ }^{c}$, Y. Minaev ${ }^{b}$, L. H. Olsen ${ }^{c}$, Y. Panebrattsev ${ }^{b}$, A. Pfeiffer $^{c}$, I. Ravinovich ${ }^{d}$, S. Razin ${ }^{b}$, P. Rehak ${ }^{f}$, A. Schön ${ }^{c}$, J. Schukraft ${ }^{g}$, M. Sampietro ${ }^{e}$, S. Shimansky ${ }^{b}$, A. Shor ${ }^{d, 2}$, J. Sokol ${ }^{d}$, H. J. Specht ${ }^{c}$, V. Steiner ${ }^{d}$, S. Tapprogge ${ }^{c}$, G. Tel-Zur ${ }^{d}$, I. Tserruya ${ }^{d}$, Th. Ullrich ${ }^{c}$, C. Voigt ${ }^{c}$, J.P. Wurm ${ }^{a}$, V. Yurevich ${ }^{a}$

${ }^{a}$ Max-Planck-Institut für Kernphysik, 69117 Heidelberg, Germany

${ }^{b}$ visiting from JINR, Dubna, Russia

${ }^{c}$ Physikalisches Institut der Universität Heidelberg, 69120 Heidelberg, Germany ${ }^{3}$

${ }^{d}$ Weizmann Institute, Rehovot 76100 , Israel ${ }^{4}$

${ }^{e}$ Politecnico di Milano, 20133 Milano, Italy

${ }^{f}$ Brookhaven National Laboratory, Upton, NY 11973, USA ${ }^{5}$

${ }^{g} \mathrm{CERN}, 1211$ Geneva 23, Switzerland

(Submitted to Physical Review Letters)

Invited Talk given at the Eleventh International Conference on Ultra-Relativistic Nucleus-Nucleus Collisions in Monterey, California, Janunary 1995

1) now at Universität Bonn, Germany

2) now at Soreq Nuclear Research Center, Yavne, Israel

3) supported by BMFT under grant 06HD525I

4) supported by Minerva, the German Israeli Foundation and the H. Gutwirth Fund

5) supported by DOE under contract DE-AC02-76CH00016 
Real and virtual photons (dileptons) are only a minute portion of what is produced during the lifetime of a nuclear fireball. In reward for the pains to measure sources so faint, and embedded in a huge background of hadrons, the promise is held by their physics potential: dileptons and direct photons are unique in that they allow to study the early collision dynamics. The argument dates back sixteen years when it was first proposed by Shuryak [1] to search for the quark-gluon plasma. As electromagnetic radiation, the mean free path is large compared to the size of the interaction system and, once produced, dileptons and photons leave freely without further interactions. The physics information attached to those penetrating probes derives from the emission rate per unit volume and unit time of electromagnetic radiation being a strongly increasing function of temperature. Dileptons and photons are therefore most abundantly produced at the early stages when temperature and energy density have their highest values.

The NA45 experiment studies the production of low-mass electron pairs and photons in nucleon-nucleus and nucleus-nucleus collisions at the CERN SPS with the Cherenkov Ring Electron Spectrometer CERES. Our goal is to receive a direct signal of hot and dense matter in the low-mass region, with some perspective that the large reservoir of partonic degrees sets its mark on $\mathrm{e}^{+} \mathrm{e}^{-}$emission. The massive electron pairs produced in the sequence of collisions of primary partons via $q \bar{q}$ (Drell-Yan), or by other QCD processes, in approach to a thermalized parton phase, are beyond the reach of CERES. Thermal pair production from the conjectured quark-gluon phase via $q \bar{q}$ annihilation might be observable only in the intermediate mass range above $1 \mathrm{GeV} / \mathrm{c}^{2}[2,3]$.

This talk reports on the results of $\mathrm{e}^{+} \mathrm{e}^{-}$-pair production in $450 \mathrm{GeV} / \mathrm{u}$ - $\mathrm{Be}$ and $\mathrm{p}$ - $\mathrm{Au}$ experiments, and on $200 \mathrm{GeV} / \mathrm{u} \mathrm{S}-\mathrm{Au}$ photon and $\mathrm{e}^{+} \mathrm{e}^{-}$pair production. The surprising result of the latter supports our optimism to grasp a sight through the freeze-out 'corona' into the fireball. The results on direct photons and dileptons are reviewed with a broader scope by I. Tserruya at this Conference [4]. The talk concludes by sketching the perspective of CERES at the SPS $\mathrm{Pb}$ beam.

\section{LEPTON PAIRS FROM NUCLEAR COLLISIONS}

Thermal radiation is our main subject of interest. In addition, dileptons as virtual photons have the property of mass that allows for the distinct measurement of vector mesons. The suppression of the $J / \Psi$ and $\Psi^{\prime}$ addressed by the NA38 collaboration and the enhancement of the $\phi /(\rho+\omega)$ ratio observed by the HELIOS/3 collaboration are prominent titles at this conference $[5,6]$. Here, we will focus on the $\rho$ meson that acts as an amplifier of thermal radiation, by some conspiracy of nature $[7,8]$.

\subsection{Thermal production and $\varrho$ meson}

Thermal production of photons and dileptons is governed by an emission rate per unit volume and unit time, and therefore measures energy densities, local in space and time. Under some simplifying assumptions, their production rate $R$ can be expressed, to lowest orders in $\alpha$ and $\alpha_{s}$, as

$$
R=d N / d^{4} x \propto \begin{cases}\alpha^{2} T^{4} & \text { for dileptons [2] } \\ \alpha \alpha_{s} T^{4} & \text { for photons [9]. }\end{cases}
$$

The total yield, the mass and $p_{\perp}$ spectra derive from integrating $R$, strongly dependent on $T$ or the local energy density, over the space-time evolution. It will scale with the square of the particle density. 
Thermal radiation from two-pion annihilation is readily accessible by CERES in the low-mass region. This QED process is modified by the pion form factor and has been proposed as a source of low-mass lepton pairs long ago [10]. In its simplest description, there will be a broad distribution that rises from threshold at $m=2 m_{\pi}$ and centers at low masses, as determined by the temperature of the medium. In the mass region of the $\rho$, however, the thermal yield receives amplification $[2,11]$ due to the $\rho$ pole in the pion form factor, mediating between the two-pion channel and the virtual photon, according to the vector dominance model. The low-mass dilepton spectrum shown in Fig. 1 displays the two-pion annihilation, along with the other processes, as calculated [12] in the Bjorken hydrodynamical model.

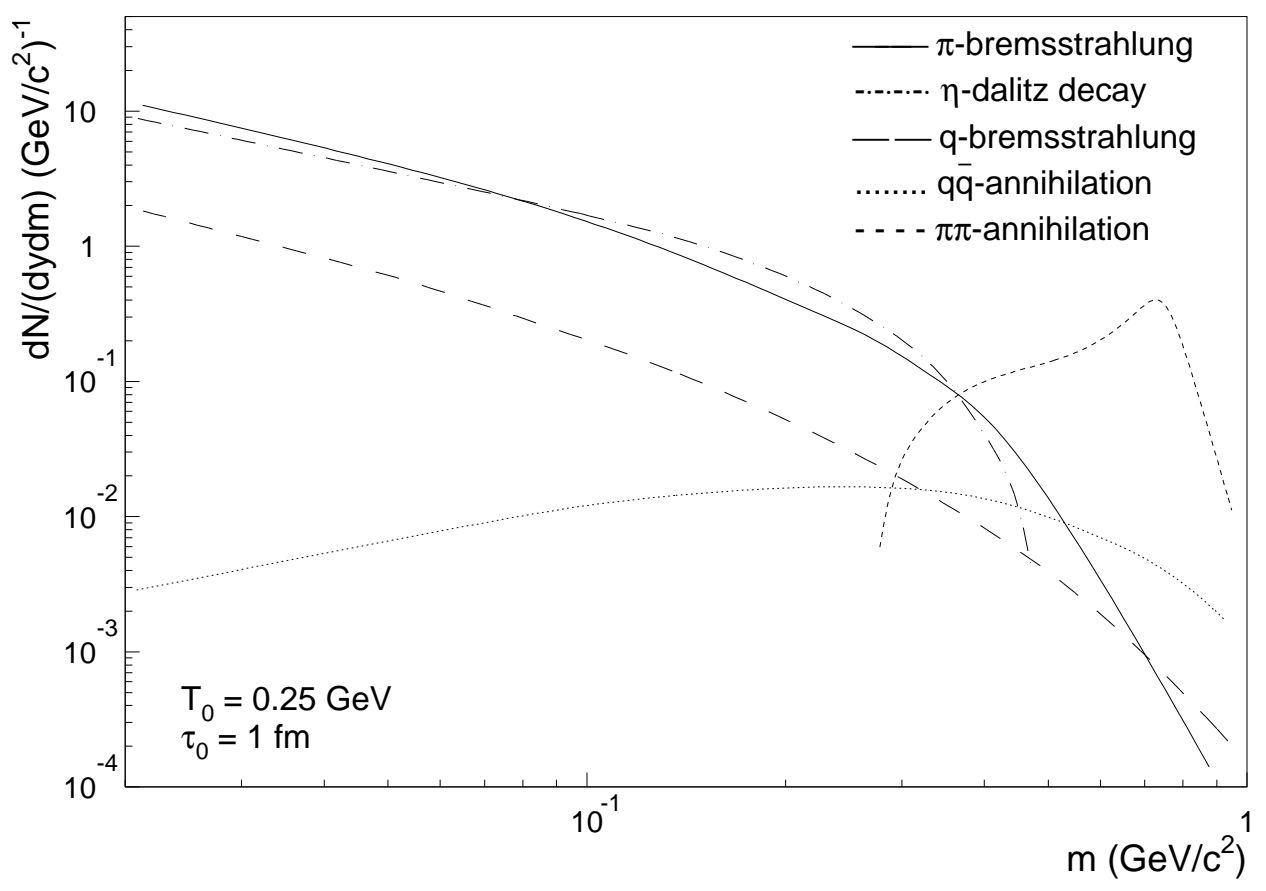

Figure 1: Various sources of dileptons at masses below $1 \mathrm{GeV} / \mathrm{c}^{2}$ [12] (initial temperature $250 \mathrm{MeV}$, formation time $1 \mathrm{fm} / \mathrm{c}$ )

To further elucidate the role of the $\rho$ meson for thermal radiation in the low-mass region, we consider a potential signal of the onset of the restoration of chiral symmetry [7]. The excitation and properties of $\rho$ mesons might be strongly modified by chiral restoration $[13,14]$. A decreasing $\rho$ mass will not only shift, but further amplify [15] the thermal radiation through the modified form factor of the pion. Due to the short life time of $\tau=$ $1.3 \mathrm{fm} / \mathrm{c}$, the decay $\rho \rightarrow \mathrm{e}^{+} \mathrm{e}^{-}$will occur within the dense matter, and the mass spectrum of electron pairs will convey the state of the medium. In hadronic observables, i.e. $\rho \rightarrow \pi \pi$, such information is lost, due to rescattering of the hadrons in dense matter.

\subsection{Hadron decays as reference signal and background}

In the absence of any new physics, dilepton and photon spectra are described by hadron decays. We like to refer to those as the conventional sources and to the yield from their decay as the reference signal. For our mass range, the hadrons include the light pseudoscalar mesons $\pi^{\circ}, \eta, \eta^{\prime}$, and the vector mesons $\rho, \omega$, and $\phi$. In the low-mass region, the dominant contributions are the Dalitz decays $\pi^{\circ}, \eta \rightarrow \mathrm{e}^{+} \mathrm{e}^{-} \gamma$ and $\omega \rightarrow \pi^{\circ} \mathrm{e}^{+} \mathrm{e}^{-}$and the direct decays $\rho / \omega \rightarrow \mathrm{e}^{+} \mathrm{e}^{-}$. The signal density scales with charged particle density. 
For invariant masses above $200 \mathrm{MeV} / \mathrm{c}^{2}$ and $p_{\perp} \geq 200 \mathrm{MeV} / \mathrm{c}$, the $\mathrm{e}^{+} \mathrm{e}^{-}$signal is of order $10^{-6} / \pi^{\circ}$, resulting in $5 \cdot 10^{-6} /$ event for $\mathrm{p}$-Be $\left(N_{c h}=3.5\right), 2 \cdot 10^{-4} /$ event for S-Au $\left(N_{c h}=150\right)$, and $7 \cdot 10^{-4} /$ event for $\mathrm{Pb}-\mathrm{Au}\left(N_{c h} \simeq 500\right)$.

In search for interesting physics, most of the reference signal is mere physics background. There is also an instrumental background, a fierce companion of $\mathrm{e}^{+} \mathrm{e}^{-}$experiments, due to external conversion $\gamma \rightarrow \mathrm{e}^{+} \mathrm{e}^{-}$in the material exposed. A very efficient rejection is mandatory: the electron (positron) tracks of unrecognized conversions give rise to the combinatorial pair background; the same applies to close Dalitz pairs.

\section{THE CERES EXPERIMENT 1992-1994}

CERES is the only heavy-ion experiment dedicated to the measurement of $\mathrm{e}^{+} \mathrm{e}^{-}$ pairs at ultra-relativistic energies. For that purpose, we have developed a novel spectrometer (see Fig. 2) that is optimized in many ways for minimum response to hadrons and photons of hadronic origin. It covers the pseudorapidity region $2.1 \leq \eta \leq 2.7$ and allows to explore the invariant mass range from $50 \mathrm{MeV} / \mathrm{c}^{2}$ to above $1.5 \mathrm{GeV} / \mathrm{c}^{2}$. The upper limit is set by statistics. A detailed description of the spectrometer is given in Ref. [17], here I restrict myself to a few essentials.

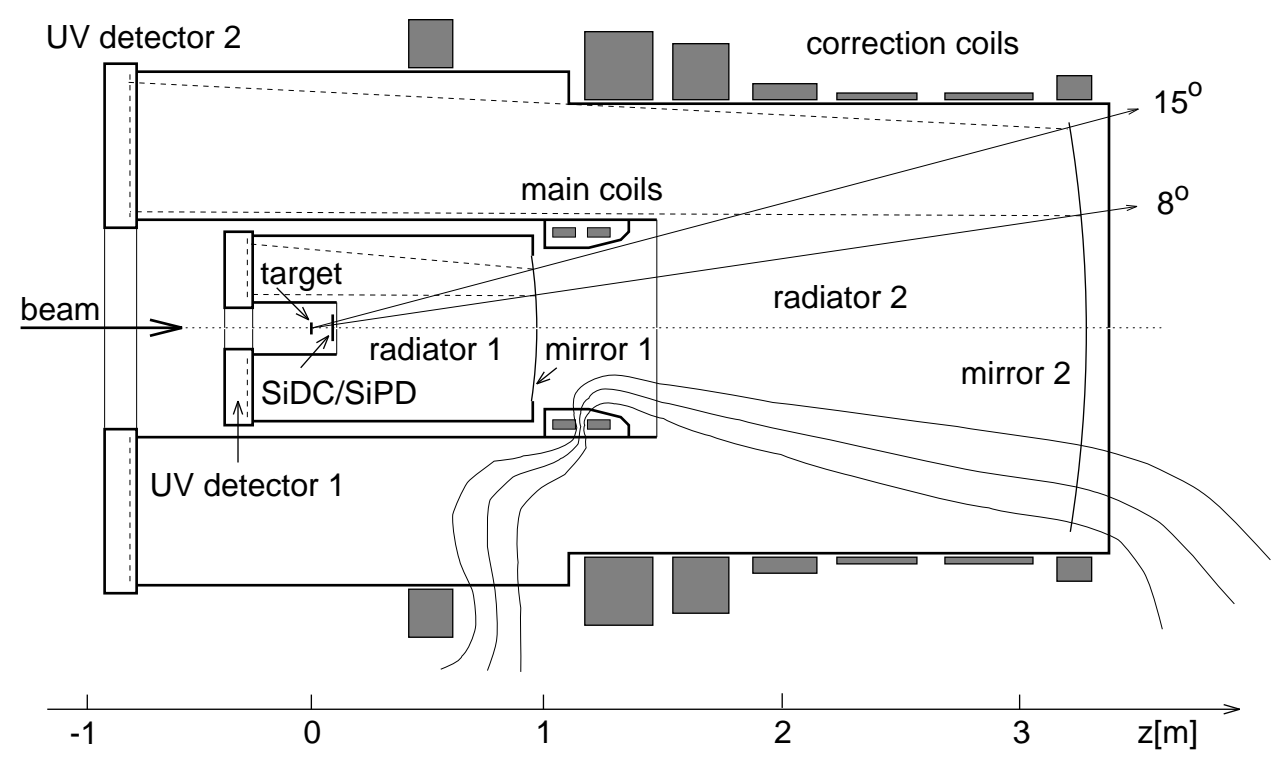

Figure 2: Schematic view of the CERES spectrometer

\subsection{Spectrometer}

Particle identification and momentum measurement are based on two azimuthally symmetric RICH detectors with a Cherenkov threshold $(\gamma \simeq 32)$ high enough to be blind for the large majority of hadrons. The magnetic field of a double superconducting solenoid is corrected by outer coils such as to provide the azimuthal deflection for momentum measurement only on a short section between the two radiators. This allows to recognize conversions as double rings in RICH-1, before they are opened by the field (Fig. 3.1). The material in the acceptance is kept to $X / X_{\circ} \leq 2.3 \%$, including the target material seen from the vertex. This is achieved with a segmented target of 30 - 40 discs of diameter small enough and spacing along the beam wide enough to expose effectively only one of them. 

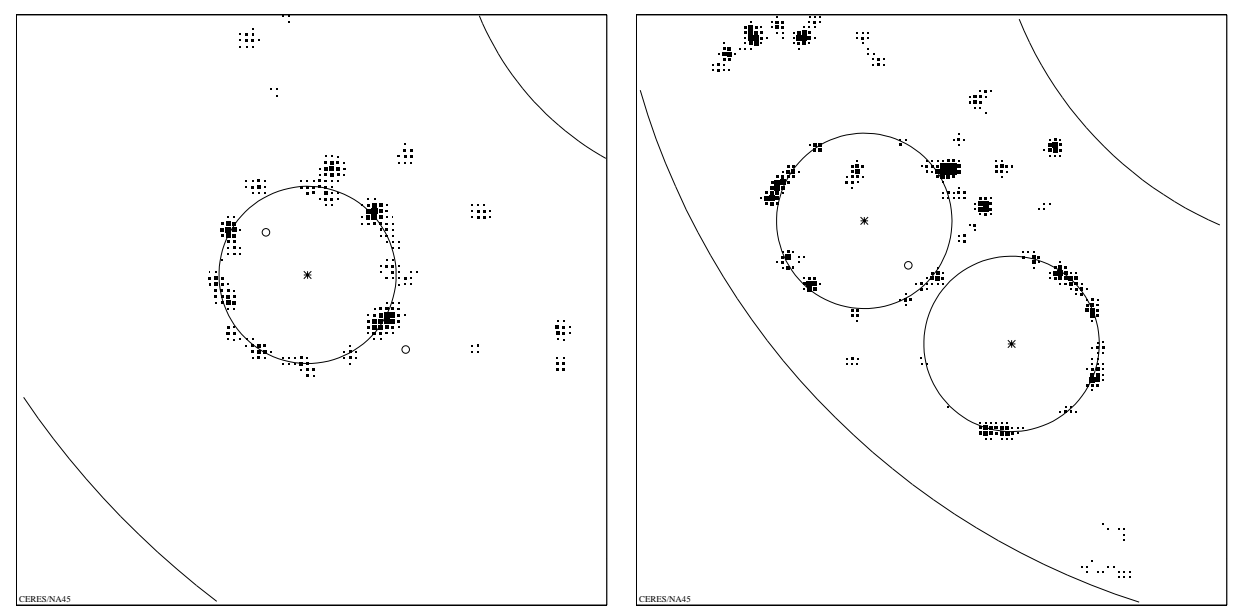

Figure 3: Photon conversion in RICH-1 (left) and RICH-2 (right) (S-Au central)

A novel radial-drift silicon detector with 360 charge-collecting anodes on the outer perimeter of a 3" wafer [18] situated closely behind the target is used for high-resolution vertex reconstruction and tracking. Its prime purpose is to supply additional rejection of photon conversions and Dalitz pairs which is necessary for a sufficient reduction of the combinatorial background in nucleus-nucleus collisions. An additional silicon detector [19] segmented into 64 pads supplies the multiplicity information for the first-level trigger.

\subsection{Data sets}

The $\mathrm{S}-\mathrm{Au}$ results presented in this talk were obtained from the analysis of data taken in the spring of 1992 . A total of $3.6 \cdot 10^{6}$ first-level and $2.7 \cdot 10^{6}$ second-level triggers were recorded. The second-level trigger recognizes Cherenkov rings in the first RICH; the decision is made by a processor array which searches for pairs of opened rings while suppressing the background of close pairs $\left(\theta_{e e} \leq 35 \mathrm{mrad}\right)[17,20]$.

The $450 \mathrm{GeV}$ p-Be and p-Au data were taken in summer 1993 when the electromagnetic calorimeter of the TAPS collaboration consisting of $384 \mathrm{BaF}_{2}$ detectors was operating downstream of CERES $(3.1 \leq y \leq 4.0)[21]$ in coincidence. The high enrichment ( $\simeq 175$ and $\simeq 75$ for $\mathrm{p}$-Be and $\mathrm{p}$ - Au, respectively) obtained with a combined intermediate and second-level trigger scheme - selecting events with at least two open rings - was essential to collect the large data samples of $2.1 \cdot 10^{9}$ and $2.7 \cdot 10^{8}$ minimum-bias equivalent events, for $\mathrm{p}$-Be and $\mathrm{p}$ - $\mathrm{Au}$, respectively.

\subsection{Data analysis}

The off-line reconstruction of electron rings is done without prior knowledge of the Cherenkov ring centers. After removing electronic noise and ionization clusters, the entire plane of pads is searched for candidate rings with asymptotic radius using a Hough point-to-ring transformation. In the vicinity of these candidates, single-photon hits are reconstructed and used to determine the ring centers by a fitting procedure. Various ring quality criteria are applied to distinguish genuine Cherenkov rings from fake rings originating from random combinations of hits. For the more demanding S-Au analysis, an artificial neural network was employed for optimizing the multidimensional decisions.

The centers of accepted rings of common polar angle $\theta$, with respect to the beam axis, are then combined to tracks. Two tracks sharing the same unresolved double ring in the first RICH detector are rejected as photon conversion candidates. 
The quality of the matching of tracks is limited by the ring center resolution and multiple scattering. The momentum resolution is still somewhat impaired by finite calibration errors affecting the track resolution; the measured mass resolution at the position of the $\rho$ is $11 \%$.

The most severe obstacle in the measurement of $\mathrm{e}^{+} \mathrm{e}^{-}$pairs is the combinatorial pair background. It arises from a large number of soft pairs which are not fully reconstructed such that single electrons remain among the track candidates and will be combined with others to fictitious pairs. We exploit, however, the fact that the inclusive electron spectrum from $\pi^{\circ}$ Dalitz decays and conversions is considerably softer than that of pairs with, say, $m \geq 0.2 \mathrm{GeV} / \mathrm{c}^{2}$. The signal-to-background ratio can therefore be significantly improved by a $p_{\perp}$ cut on single electrons, as well as on the opening angle (close-ring cut), and on the total ring amplitude in the first RICH (double-ring cut). For S-Au, the hit information from the silicon drift detector (coordinates and energy loss amplitude) had to be employed to substantially improve the rejection of fake tracks, by requiring a common vertex, and of close electron pairs by their double-hit signature in silicon.

The remaining combinatorial background in the $\mathrm{e}^{+} \mathrm{e}^{-}$sample is determined by the like-sign pairs, as the geometrical mean of the numbers $N_{++}$and $N_{--}$. The pair signal S is then defined as $S=N_{+-}-2 \sqrt{N_{++} N_{--}}$.

\section{SYSTEMATIC STUDY OF MESON $\mathrm{e}^{+} \mathrm{e}^{-}-$DECAYS IN p-Be, p-Au}

The proton run in 1993 was performed to clarify a 10-year old controversy, whether all $\mathrm{e}^{+} \mathrm{e}^{-}$pairs in the mass range below the $\rho / \omega$ result from hadron decays. Recently, after an extensive study of $450 \mathrm{GeV}$ p-Be, HELIOS/1 arrived at such conclusion [22]. However, the margin of uncertainty for any unconventional source of $\mathrm{e}^{+} \mathrm{e}^{-}$pairs (what had been termed the 'anomalous' pair continuum) was left at $40 \%$ of the inclusive $\mathrm{e}^{+} \mathrm{e}^{-}$yield. CERES/TAPS will improve on HELIOS-1 by having collected a sample of significantly larger size that will allow not only to measure the Dalitz decays of $\eta$ (and $\eta^{\prime}$ ) with much better precision, but also to reconstruct the $\omega$ Dalitz decays - the major unknowns in the hadronic contribution.

\subsection{Results on inclusive $\mathrm{e}^{+} \mathrm{e}^{-}$pairs and comparison to hadronic sources}

First, I like to present our inclusive data on $450 \mathrm{GeV}$ p-Be and p-Au which consist of $5760 \pm 184(1126 \pm 100)$ reconstructed $\mathrm{e}^{+} \mathrm{e}^{-}$pairs, respectively, after subtraction of the combinatorial background. The signal-to-background ratio was $1 / 2.2(1 / 4.5)$. The mass spectra shown in Fig. 4 display the pair density per charged particle density within the acceptance $2.1 \leq \mathrm{y} \leq 2.65$. The average charged particle densities for the sample of reconstructed events are quoted in the figure. The statistical errors are marked by bars; the brackets reflect the systematic uncertainties in the reconstruction efficiency, the acceptance, and the trigger enhancement, and have been all added linearly to the statistical errors.

The various contributions from hadron decays, shown in the figure, have been calculated with a Monte Carlo event generator [23]. For pair masses below $140 \mathrm{MeV} / \mathrm{c}^{2}$, the $\pi^{\circ}$ Dalitz decay dominates the spectra, despite its strong suppression by cuts on $p_{\perp} \geq 50 \mathrm{MeV} / \mathrm{c}$ of the tracks, and on pair opening angle $\Theta_{e e} \geq 35 \mathrm{mrad}$. At higher masses, the Dalitz decays $\eta \rightarrow \mathrm{e}^{+} \mathrm{e}^{-} \gamma$ and $\omega \rightarrow \mathrm{e}^{+} \mathrm{e}^{-} \pi^{\circ}$ are most significant.

We assume that the invariant cross sections $d^{3} \sigma / d^{2} p_{\perp} d y$ factorize in $p_{\perp}$ and y. Both, the $p_{\perp}$ and y-distribution, were generated taking into account the observed differences between nucleon-nucleon and nucleon-nucleus interactions. The $p_{\perp}$-distributions were gener- 

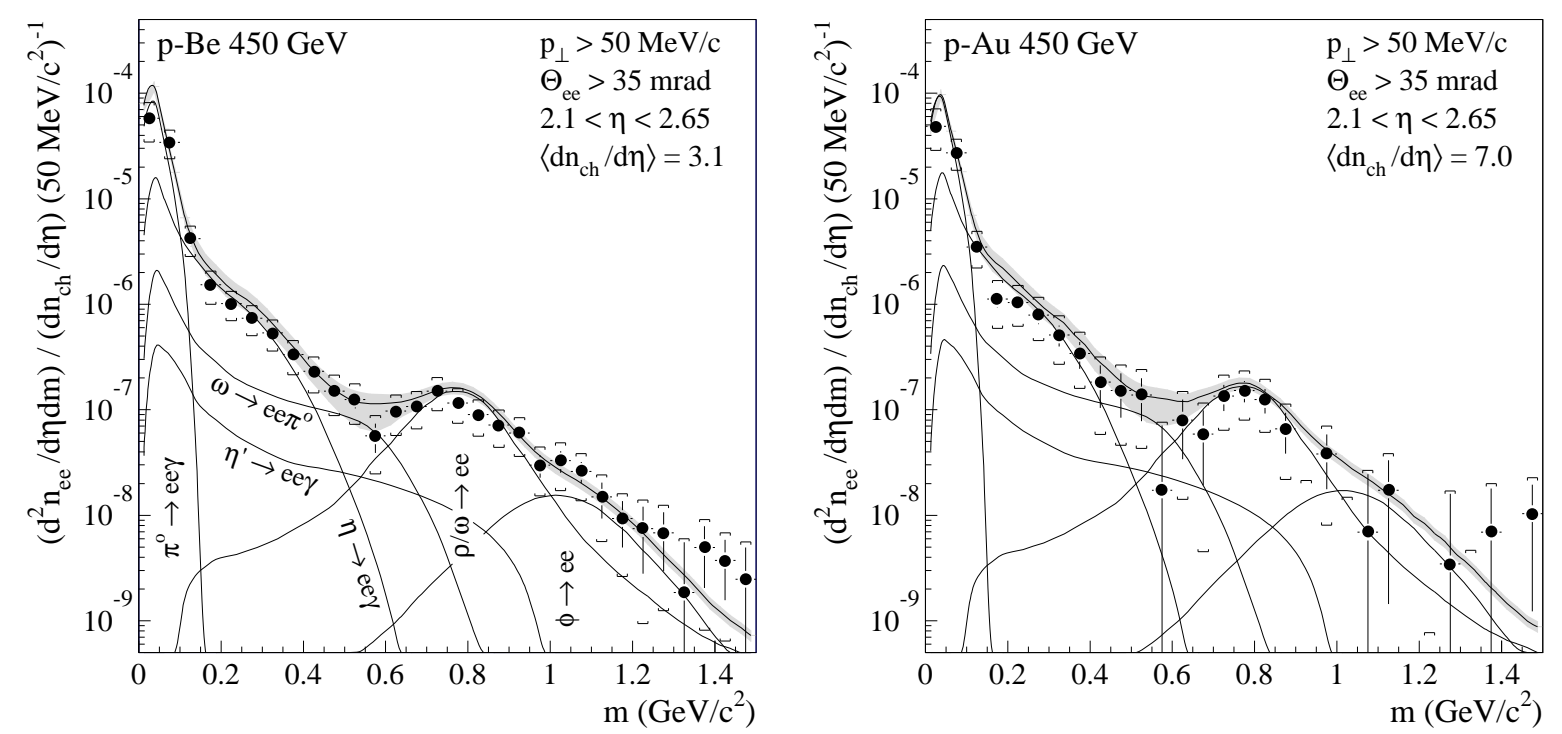

Figure 4: Mass spectra of inclusive $\mathrm{e}^{+} \mathrm{e}^{-}$pairs in $450 \mathrm{GeV}$ p-Be (left) and p-Au (right) showing the data (full circles) and the various contributions from hadron decays. The shaded region indicates the systematic error on the summed contributions.

ated assuming $m_{\perp}$-scaling [24] based on pion $p_{\perp}$-spectra from several experiments $[25,26]$. The rapidity distribution was a fit to measured data [27]. The relative rapidity densities for the various mesons were taken from ref. [28]. All Dalitz decays were treated according to the Kroll-Wada expression with the experimental transition form factors taken from ref. [29]; the vector meson decays were generated using the expressions derived by Gounaris and Sakurai in ref. [30]. Charm production and bremsstrahlung were not taken into account since they are likely to be negligible in this mass range. Finally, the laboratory momenta of the electrons were convoluted with the experimental resolution and acceptance. Predictions and data are not corrected for pair acceptance, i.e. for the loss of acceptance due to finite opening of the electron tracks which increases with mass. The uncertainties of the summed contributions are indicated by shaded regions in Fig. 4.

The $\mathrm{p}$-Be data are well reproduced by hadron decays, within our present systematic errors of $40 \%$, which confirms the conclusion drawn by Helios/1. That this remains valid also for $\mathrm{p}-\mathrm{Au}$ collisions is a new result that might deserve further attention in context of the $\mathrm{S}-\mathrm{Au}$ results.

\section{2 $\mathrm{e}^{+} \mathrm{e}^{-} \gamma$ in CERES/TAPS}

A significant fraction of the uncertainties of the previous comparison lie in the poorly known contributions of the $\eta, \omega$, and $\eta^{\prime}$ Dalitz decays. As can be seen by inspection of Fig. 4, they account for most of the yield in the $400-600 \mathrm{MeV} / \mathrm{c}^{2}$ mass range. CERES/TAPS has directly measured those decays and will improve the data on production cross sections, $p_{\perp}$ distributions and branching ratios. In addition, we foresee a new measurement of the electromagnetic transition form factors of the $\omega$, besides that of $\eta$ and $\eta^{\prime}$, which is overdue 15 years after the Lepton-G collaboration reported data that grossly overshoot the expectation of vector dominance [29]. The compounded effect of these data should significantly lower the present uncertainty of $40 \%$.

The analysis of the data is coming forth. In Fig. 5 we demonstrate the reconstruction of $\pi^{\circ}$ and $\eta$ from the $\mathrm{e}^{+} \mathrm{e}^{-} \gamma$ coincidence data. The analysis of the $\mathrm{e}^{+} \mathrm{e}^{-} \gamma \gamma$ coincidence data to reconstruct the $\omega$ is in progress. 

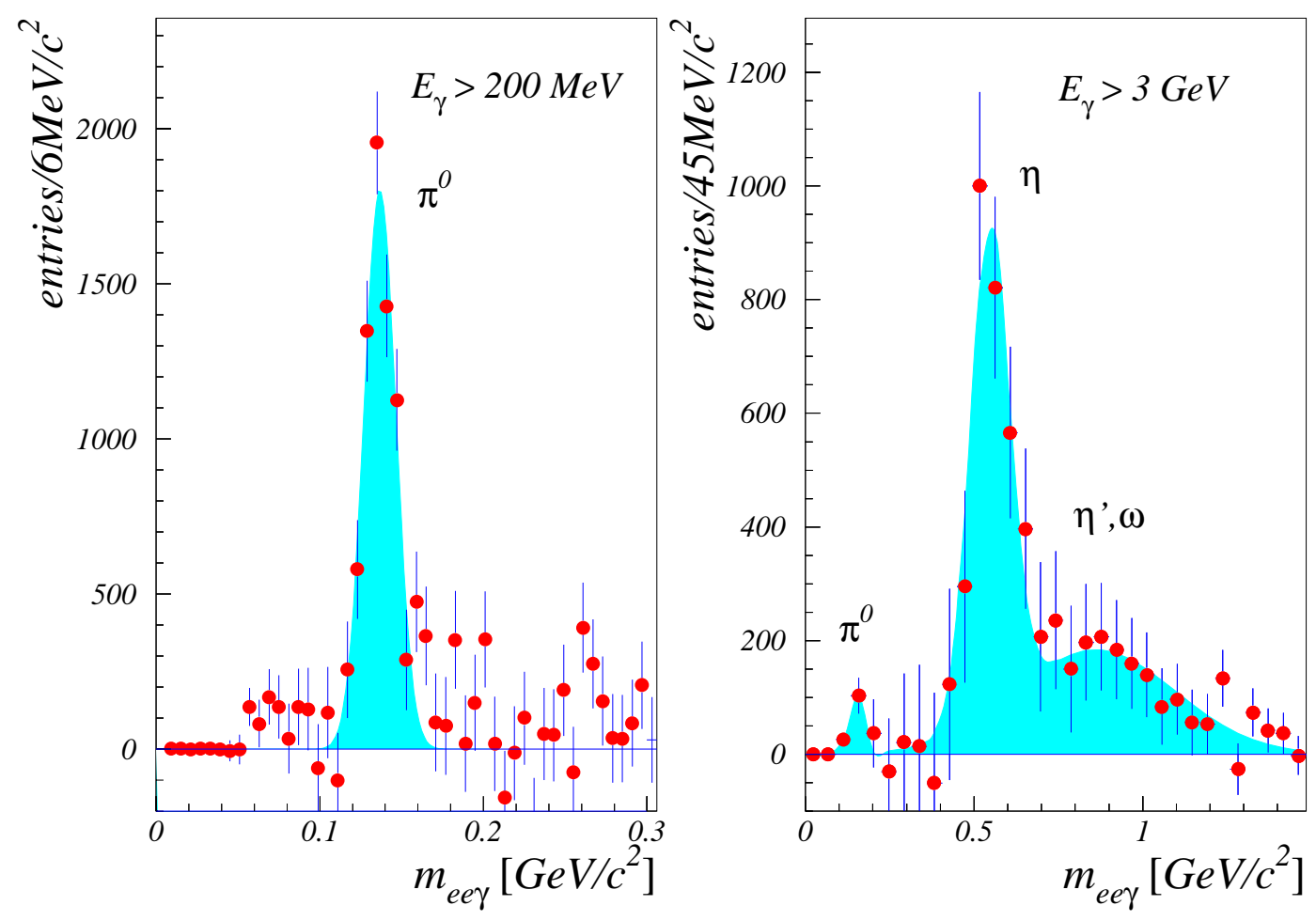

Figure 5: Preliminary spectra of reconstructed invariant mass $m_{e e \gamma}$ of the Dalitz decays $\pi^{\circ} \rightarrow \mathrm{e}^{+} \mathrm{e}^{-} \gamma$ (left) and $\eta \rightarrow \mathrm{e}^{+} \mathrm{e}^{-} \gamma$ (right) after subtraction of the combinatorial background. The accumulation of entries above the $\eta$ is dominated by $\omega \rightarrow \mathrm{e}^{+} \mathrm{e}^{-} \pi^{\circ}$ Dalitz decays incompletely reconstructed. Cuts for the $\mathrm{e}^{+} \mathrm{e}^{-}$pairs were those applied to the inclusive data; reduction of the combinatorial background in the mass region of the $\eta$ was accomplished by rejecting showers with energy less than $3 \mathrm{GeV}$ in TAPS. The shower analysis has been done by the Giessen University group [31].

\section{SULPHUR-GOLD COLLISIONS}

\subsection{Real Photons}

The capability of the CERES spectrometer to recognize and reject photon conversions was employed for the S-Au data to study photon production by the conversion method. Preliminary results were presented already at Quark Matter '93 [32]. I report here on a reanalysis of these data, surely not to arouse excitement. For one, we wish to give our best to the topic of direct photons; for the other part, the tedious assessment of the hadronic source contributions is done once, and on a consistent basis, for $\mathrm{p}$-Be to $\mathrm{S}-\mathrm{Au}$, for photons and electron pairs.

The conversions on the target and other material in the acceptance $\left(2.3 \% X / X_{\circ}\right)$ are recognized as a $\mathrm{V}$-shaped pair of tracks with a common ring in the first RICH and a minimum opening angle of $8 \mathrm{mrad}$ in the second. Only tracks with a maximum $\varphi$-deflection corresponding to $p_{\perp} \geq 50 \mathrm{MeV} / \mathrm{c}$ are accepted. A total of $8.5 \cdot 10^{5} \mathrm{~S}$ - Au collisions selected by two different centrality triggers have been analyzed. Out of these, $9.5 \cdot 10^{4}$ conversions were reconstructed.

In order to evaluate the photon $p_{\perp}$ spectrum, the reconstruction efficiency and the smearing due to the instrumental momentum resolution has to be applied. The raw $p_{\perp}$ spectrum is contaminated by $\pi^{\circ}$ Dalitz decays that are not resolved by our limited double-ring resolution of $\simeq 8$ mrad. Their fraction amounts to $18 \%$ of the inclusive photon 


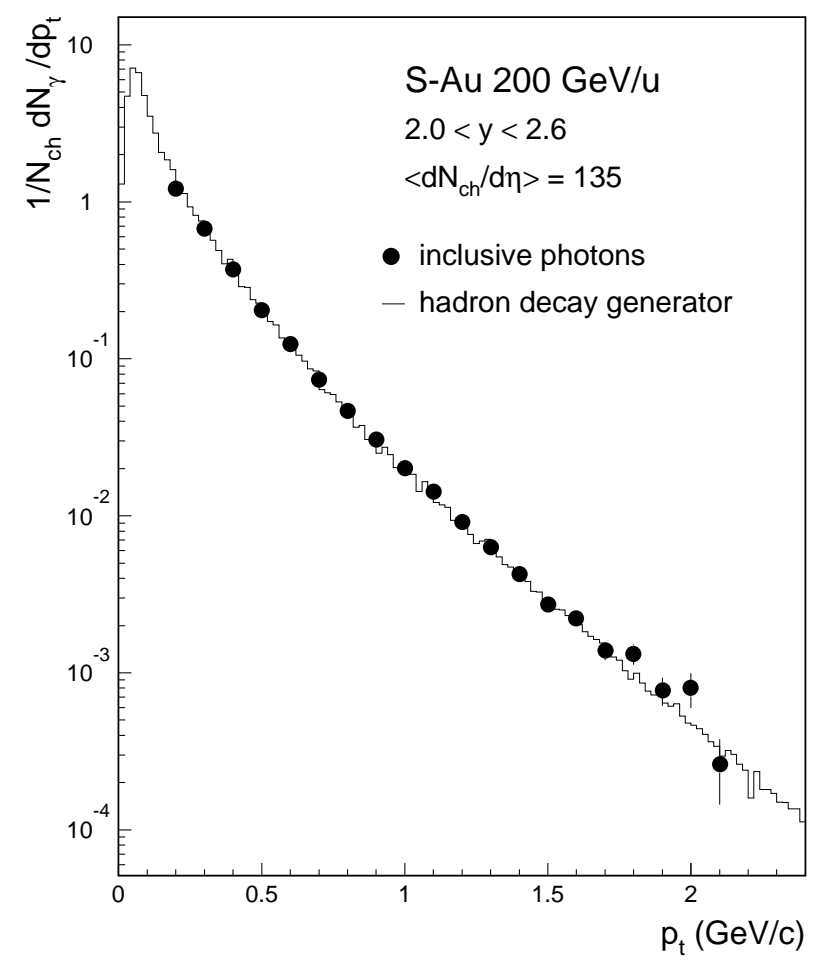

Figure 6: $p_{\perp}$ spectrum of photons from central S-Au collisions, normalized to $N_{c h}$. Shown is the inclusive measurement with statistical errors in comparison to the expectation from hadron decays.

spectrum, and is nearly independent of $p_{\perp}$. This contribution is modeled with a MonteCarlo simulation, and subtracted from the spectrum.

The resulting $p_{\perp}$ spectrum of photons measured in the rapidity interval $2.0 \leq y \leq$ 2.6 for central S-Au collisions is shown in Fig. 6. The photon yield is normalized to the number of charged particles in the acceptance (corrected for $\delta$ rays and conversions). The average charged particle density of the reconstructed photon sample is 135 . The contribution of all known hadron decays is also shown. It is dominated by $\pi^{\circ}, \eta \rightarrow \gamma \gamma$ at low $p_{\perp}$; all meson decays up to the $\phi$ are included. The Monte Carlo simulation is essentially the same as for $\mathrm{p}$-Be, but takes into account the changed $d N / d y$-distribution and the slightly modified $p_{\perp}$-spectrum in S-Au collisions $[33,34,35,36]$.

Lacking accurate data for $\pi^{\circ} / N_{c h}$ for sulphur on heavy targets, the required normalization of the generated photon yield to the measured charged particle density was based on the value $\left(d \sigma_{\pi^{\circ}} / d \eta\right)\left(d \sigma_{N_{c h}} / d \eta\right)=0.46$, measured in p-p at $y_{c m}=0$ [28]. A slightly reduced value of $\pi^{\circ} / N_{c h}=0.44$ was adopted for the simulations, in order to account for an increase in baryon density at mid-rapidity in $\mathrm{S}-\mathrm{Au}$, as compared to $\mathrm{p}-\mathrm{p}$. This correction was made by extrapolating S-Ag data [37] to S-Au; these data from NA35 also justify our assumption of constant particle composition from $p-p$ to sulphur on heavy targets.

The shape as well as the absolute yield of the $p_{\perp}$ distributions are well described by the known hadronic sources, clearly visible in Fig. 6. To quantify this result, we quote the integral photon yield per charged particle in the range $0.4 \leq p_{\perp} \leq 2.0 \mathrm{GeV} / \mathrm{c}$

$$
r_{\gamma}=\frac{1}{N_{c h}} \int \frac{d N_{\gamma}}{d p_{\perp}} d p_{\perp}
$$

Integrated over the full phase space, $r_{\gamma} \simeq 1$ for photons from hadron decays. For our 
window in rapidity and $p_{\perp}$ the value is reduced to 0.09 . Defining the yield of direct photons as the surplus of the measured over the yield expected from hadron decays, we obtain

$$
\frac{r_{\text {dir }}}{r_{\text {hadr }}}=\frac{r_{\text {data }}}{r_{\text {hadr }}}-1=1_{-11.5}^{+7.5} \% .
$$

Data and simulation contribute about equally to the uncertainties quoted which are entirely of systematic origin. A detailed assessment of the systematic errors results in an upper limit for $r_{d i r} / r_{h a d r}$ of $10 \%$ with $90 \%$ confidence limit. Within this error margin, the photon spectra are accounted for by hadron decays.

\section{$5.2 \quad \mathrm{e}^{+} \mathrm{e}^{-}$-Pairs}

We present here the results on low-mass electron pairs from S-Au central collisions [38]. The centrality was defined by two trigger levels on $d N_{c h} / d y$ (measured by the silicon pad detector), at thresholds of 140 and 50, combined with weights 4 and 1, respectively, resulting in $\left\langle d N_{c h} / d y\right\rangle=125$. Due to the higher combinatorial background in sulphurinduced collisions $\left(S / B \propto 1 / N_{c h}\right)$, the $p_{\perp}$ cut was raised to $0.2 \mathrm{GeV} / \mathrm{c}$.

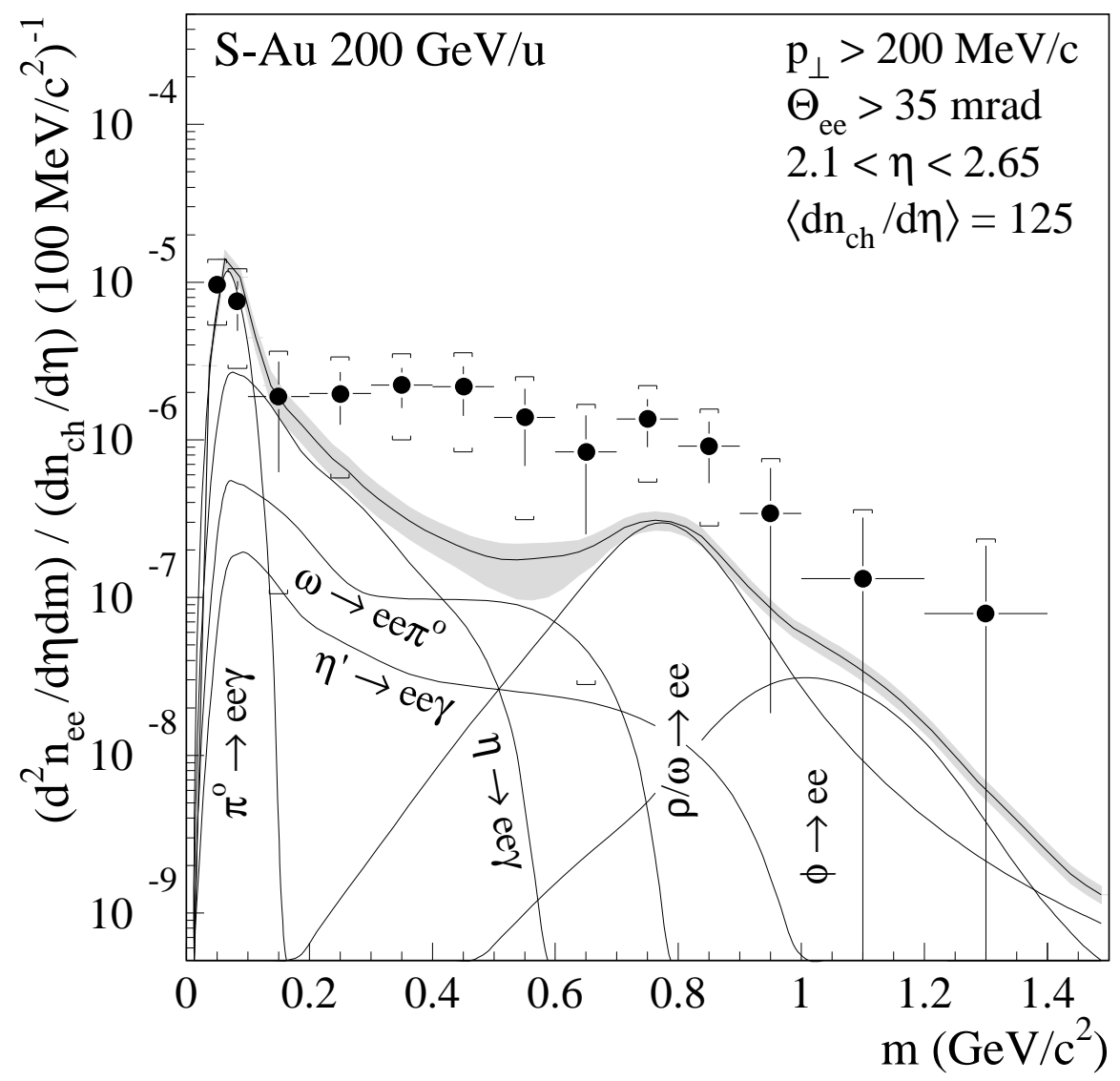

Figure 7: Inclusive $\mathrm{e}^{+} \mathrm{e}^{-}$mass spectra in central $200 \mathrm{GeV} / \mathrm{u} \mathrm{S}-\mathrm{Au}$ collisions

The final sample for $m \geq 0.2 \mathrm{GeV} / \mathrm{c}^{2}$ consists of 4249 pairs of which 2346 are $\mathrm{e}^{+} \mathrm{e}^{-}$. The resulting net pair signal is $445 \pm 65$ with a $\mathrm{S} / \mathrm{B}$ ratio of $1 / 4.3$. The inclusive mass spectrum is shown in Fig. 7. As in Fig. 4, the pair density per charged particle density within the pseudo-rapidity acceptance $2.1 \leq y \leq 2.65$ is plotted. The statistical errors are marked by bars; the brackets reflect the systematic uncertainties due to the reconstruction 
efficiency, acceptance and trigger enhancement, and are linearly added to the statistical errors.

The Monte Carlo simulation of the various contributions of hadron decays, described at some length before, is also shown in Fig. 7. Both the simulated spectrum and the data are not corrected for pair acceptance. This correction would require deeper knowledge of all sources of pairs which we are lacking for the S-Au case.

We observe that at very low masses $m \leq 200 \mathrm{Mev} / \mathrm{c}^{2}$, the data are well reproduced by the simulation. This is not astonishing since $\pi^{\circ}$ and $\eta$ Dalitz decays flood this part of the spectrum. Above, the mass spectrum has a different shape and the observed yield is much larger than the one predicted from hadron decays. The excess starts at masses of about $200 \mathrm{MeV} / \mathrm{c}^{2}$ and persists up to the highest masses covered in the experiment above the $\rho / \omega$ meson. The enhancement factor, defined as the ratio of the integral of the data over the integral of the predicted sources, is found to be $5.0 \pm 0.7$ (stat.) \pm 2.0 (syst.) in the mass region $0.2<m<1.5 \mathrm{GeV} / \mathrm{c}^{2}$.

If the observed excess originates from thermal pair radiation, it should vary as the square of the charged particle density. Our S-Au data sample is not sufficiently large to allow for a differential study of the excess. We might, however, compare with the results obtained by the HELIOS / 3 collaboration, which also show an excess of lepton pairs in the low and intermediate mass region [6]. We have worked out from their preliminary data in the comparable mass region an enhancement factor of $\simeq 1.6$, as the ratio of the $\mu^{+} \mu^{-}$ density per charged particle for $\mathrm{S}-\mathrm{W}$ over that for $\mathrm{p}-\mathrm{W}$. As far as the source of the excess lepton pairs scales linearly with particle density, the enhancement factors should be the same; if it scales quadratically, they should be proportional to the accompanying particle densities. We stress that quadratic scaling is on safe grounds here, since the collision systems are nearly identical, and both experiments trigger on central collisions implying similar transverse extensions of the sources. In the acceptance of CERES $(2.1 \leq y \leq 2.65)$, central collisions lead to particle densities larger by a factor 2 to 3 than in the more forward acceptance of HELIOS $/ 3(3.9 \leq y \leq 5.8)$.

This comparison of the two data sets favors a quadratic dependence. Together with the onset of the excess at $m_{e e} \simeq 2 m_{\pi}$ and the persistence of the enhancement in the $\rho$ mass region, these signatures are suggestive of an explanation by pion annihilation.

\section{PERSPECTIVE FOR SPS LEAD BEAM EXPERIMENTS}

The CERES spectrometer has been continually upgraded since its first physics data taking in 1992, and the performance in data quality and electronic read-out speed has dramatically improved compared to the time of the sulphur run. A decisive step was put forward towards SPS $\mathrm{Pb}$ beam experiments by introducing external tracking before the first RICH detector, using a vertex telescope of silicon drift detectors. The $\mathrm{Pb}$ test run in fall 1994 has clearly established that the upgraded spectrometer can cope with the highest multiplicities in central $\mathrm{Pb}-\mathrm{Au}$ collisions [39].

With a newly developed data acquisition system, we expect from a sample of central triggers $\mathrm{dN}_{c h} / \mathrm{dy} \geq 400$ that can be collected within a month of running time to reconstruct a reference signal of several thousand hadron decays for a $p_{\perp}$ cut of $0.2 \mathrm{GeV} / \mathrm{c}^{2}$. Adding conservatively the direct source of low-mass pairs, we expect a sample of $\simeq 10^{4}$. The high accuracy that evidently can be reached for the low masses will allow to study the $p_{\perp}$ distribution and the multiplicity dependence of the excess, key questions that could not be addressed by the limited statistics of the S-Au sample. CERES with its acceptance coverage close to mid-rapidity is well suited for this study. 
CERES has measured $\mathrm{e}^{+} \mathrm{e}^{-}$pairs and photons with a dedicated Cherenkov spectrometer located close to mid-rapidity.

In a systematic study of $450 \mathrm{GeV}$-Be, meson decays are found to fully account for the inclusive low-mass $\mathrm{e}^{+} \mathrm{e}^{-}$spectrum measured with high statistical accuracy, within systematic errors of $40 \%$. This remains valid for $\mathrm{p}$-Au. The analysis of the direct measurement of the $\eta$ and $\omega$ Dalitz decays is expected to significantly reduce systematic errors concerning cross sections, branching ratios and form factors.

No direct photons have been observed in $200 \mathrm{GeV} / \mathrm{u} \mathrm{S}-\mathrm{Au}$, within a limit of $10 \%$ $(90 \% \mathrm{CL})$, in the range $0.4 \mathrm{GeV} / \mathrm{c} \leq p_{\perp} \leq 2.0 \mathrm{GeV} / \mathrm{c}$.

In central $200 \mathrm{GeV} / \mathrm{u}$ S-Au collisions, $\mathrm{e}^{+} \mathrm{e}^{-}$pairs exhibit an enhancement over hadronic sources of $5.0 \pm 0.7$ (stat.) \pm 2.0 (syst.) in the mass region $0.2<m<1.5 \mathrm{GeV} / \mathrm{c}^{2}$. The shape of the excess, its onset at $2 m_{\pi}$, and its particle-density dependence compatible with a quadratic one are all suggestive of two-pion annihilation as the origin. This would imply that we have received a signal of thermal radiation from the hot and dense interacting matter. We are looking forward to further investigate this new source of low-mass electron pairs and study its evolution under the conditions offered by the $\mathrm{Pb}$ beam.

\section{References}

[1] E.V. Shuryak, Phys. Lett. 78B (1978) 150

[2] K. Kajantie et al., Phys. Rev. D34 (1986) 2746

[3] P.V. Ruuskanen, Quark-Gluon-Plasma,

[4] I. Tserruya, these Proceedings

[5] S. Ramos for the NA38 Collaboration, these Proceedings

[6] M. Masera for the HELIOS/3 collaboration, these Proceedings R. Hwa (ed.), World Scientific, Singapore 1990

[7] R. Pisarski, Phys. Lett. B110 (1982) 155

[8] G.E. Brown, Progr. Theor. Phys. 91 (1987) 85

[9] M. Neubert, Zeit. Phys. C42 (1989) 231

[10] T. Goldman, Minh Duong-van, R. Blankenbecler, Phys. Rev. D20 (1979) 619

[11] C. Gale and J. Kapusta, Phys. Rev. C35 (1987) 2107

[12] J. Cleymans, V.V. Golovizinin, and K. Redlich, Zeit. Phys. C59 (1993) 495

[13] V. Bernard et al., Phys. Lett. 227B (1989) 465

[14] G.E. Brown et. al., Phys. Rev. C43 (1991) 1881

[15] G.Q. Li and C.M. Ko, Nucl. Phys. A582 (1995) 731

[16] P.V. Ruuskanen in Quark Gluon Plasma Signatures, V. Bernard et al. (eds.), Editions Frontieres, Gif-sur-Yvette 1991

[17] R. Baur et al., Nucl. Instr. Meth. A343 (1994) 87

[18] W. Chen et al., IEEE Trans. Nucl. Sci. 39 (1992) 619

[19] T.F. Günzel et al., Nucl. Instr. Meth. A316 (1992) 259

[20] J. Gläß et al., IEEE Trans. Nucl. Sci. 37 (1990) 241

[21] P. Rehak et al., CERN/SPSLC 92-48, SPSLC/P237 Add 2., together with TAPS Collaboration (Univ. Gießen, KVI Groningen and GSI Darmstadt)

[22] T. Åkesson et al., CERN-PPE/94-140, submitted to Zeit. Phys. C

[23] Genesis Code, T. Ullrich and D. Irmscher, unpublished

[24] M. Bourquin and J.M. Gaillard, Nucl. Phys. B114 (1976) 334

[25] B. Alper et al., Nucl. Phys. B100 (1975) 237

[26] D. Anterasyan et al., Phys. Rev. D19 (1979) 764 
[27] C. De Marzo et al., Phys. Rev. D26 (1982) 1019

[28] M. Aguilar-Benitez et al., Zeit. Phys. C50 (1991) 405

[29] L.G. Landsberg, Phys. Rep. 128 (1985) 301

[30] G.J. Gounaris and J.J. Sakurai, Phys. Rev. Lett. 21 (1966) 244

[31] M. Franke, M. Noll, W. Kühn, and V. Metag, GSI Annual Report 1994

[32] D. Irmscher, Nucl. Phys. A566 (1994) 347c

[33] T. Albrecht et al., Zeit. Phys. C55 (1992) 539

[34] T. Akesson et al., Zeit. Phys. C46 (1992) 361

[35] T. Alber et al., Nucl. Phys. A566 (1994) 35c

[36] R. Santo et al., ibidem, 61c

[37] D. Röhrich et al., ibidem 35c

[38] G. Agakichiev et al., preprint CERN-PPE/95-26 and Phys. Rev. Lett. , in print

[39] CERES report, CERN/SPSLC 95-2 